\title{
Geometric Aspects of Self-adjoint Sturm-Liouville Problems
}

\author{
Yicao Wang \\ Department of Mathematics, Hohai University, Nanjing 210098, China
}

\begin{abstract}
In the paper, we use $\mathrm{U}(2)$, the group of $2 \times 2$ unitary matices, to parameterize the space of all self-adjoint boundary conditions for a fixed Sturm-Liouville equation on the interval $[0,1]$. The adjoint action of $\mathrm{U}(2)$ on itself naturally leads to a refined classification of self-adjoint boundary conditions-each adjoint orbit is a subclass of these boundary conditions. We give explicit parameterizations of those adjoint orbits of principal type, i.e. orbits diffeomorphic to the 2-sphere $S^{2}$, and investigate the behavior of the $n$-th eigenvalue $\lambda_{n}$ as a function on such orbits.

Keywords: regular Sturm-Liouville problem, space of self-adjoint boundary conditions, adjoint orbit, eigenvalue

2008 MSC: 53D18, 53D05, 53C15
\end{abstract}

\section{Introduction}

Unbounded self-adjoint (SA for brevity) operators are very important objects in mathematical physics. In quantum mechanics, an observable is represented by an SA operator, rather than a symmetric one. In perturbative quantum field theory, when calculating the contribution of a one-loop graph, one should obtain the (regularized) determinant of a differential operator, but before that, a suitable SA extension should be chosen first. However, generally, there may be too many SA extensions by prescribing different SA boundary conditions. For example, consider the classical Sturm-Liouville

Email address: yicwang@hhu.edu.cn (Yicao Wang) 
(S-L) equation on $J=[0,1]$ :

$$
l y:=-\left(p y^{\prime}\right)^{\prime}+q y=\lambda y, \quad 0<p \in C^{1}(J), q \in C(J) .
$$

Then the set $\mathcal{U}$ of all complex SA boundary conditions can be divided into two mutually exclusive subsets. The first, called separated, includes boundary conditions of the form

$$
\left\{\begin{array}{l}
y(0) \cos \alpha-\left(p y^{\prime}\right)(0) \sin \alpha=0, \\
y(1) \cos \beta-\left(p y^{\prime}\right)(1) \sin \beta=0,
\end{array}\right.
$$

where $\alpha \in[0, \pi), \beta \in(0, \pi]$; The second, called coupled, includes boundary conditions of the form

$$
\left(\begin{array}{c}
y(1) \\
\left(p y^{\prime}\right)(1)
\end{array}\right)=e^{i \varphi} K\left(\begin{array}{c}
y(0) \\
\left(p y^{\prime}\right)(0)
\end{array}\right)
$$

where $K \in \operatorname{SL}(2, \mathbb{R})=:\left\{k=\left(\begin{array}{ll}k_{11} & k_{12} \\ k_{21} & k_{22}\end{array}\right) ; k_{i j} \in \mathbb{R}, \operatorname{det} K=1\right\}$, and $\varphi \in$ $[0,2 \pi)$.

It is well-known that the eigenvalues of the above S-L problem consisting of Eq. (1.1) and an SA boundary condition are bounded from below and can be ordered to form a non-decreasing sequence

$$
-\infty<\lambda_{0} \leq \lambda_{1} \leq \lambda_{2} \leq \cdots \leq \lambda_{n} \leq \cdots,
$$

approaching $\infty$ so that the number of times an eigenvalue can appear equals to its (geometric) multiplicity.

In this paper, we mainly put emphasis on the structure of $\mathcal{U}$ and assume that $p \equiv 1$ and $q \in C(J)$ for brevity, though the main body of results here holds for more general $p, q$ 's.

It's of interest to consider these $\lambda_{n}$ as functions on $\mathcal{U}$ and explore how they change when the boundary condition varies. It is already clear that, $\lambda_{n}$ are not continuous on $\mathcal{U}$ equipped with the natural topology [4]. However, when restricted on certain subset $S \subset \mathcal{U}, \lambda_{n}$ may have nice properties. For example, if on $S \lambda_{0}$ is bounded from below, then all these $\lambda_{n}$ are continuous on $S$. This is called the continuity principle in [4], which we shall use frequently in the following.

An abstract theorem of von Neumann implies that $\mathcal{U}$ is globally parameterized by $\mathrm{U}(2)$, the unitary group in complex dimension 2 , but in the 
mathematical literature on S-L problems, in terms of boundary data, $\mathcal{U}$ is often viewed as a set of equivalence classes of matrices, say, a submanifold of the Grassmanian of 2-dimensional subspaces in $\mathbb{C}^{4}$. In this context, (1.2) and (1.3) are in fact preferred representative of these classes and the underlying group $U(2)$ cannot be seen directly in this manner. Recently it is found in [1] that there is, more or less, a canonical way to identify $\mathcal{U}$ with $\mathrm{U}(2) \mathbb{1}$, which is the starting point of our paper.

As a smooth 4-manifold, $\mathrm{U}(2)$ is very special. It is a compact Lie group and has a rich geometry. In this paper, however, we mainly consider one aspect of this geometry and its interplay with S-L problems: $\mathrm{U}(2)$ acts on itself by conjugation, i.e. $g \cdot u=g u g^{-1}$ for $g, u \in \mathrm{U}(2)$. Orbits of this action are called adjoint orbits, each characterized by its eigenvalues (matrices in an orbit all have the same eigenvalues). Topologically, these orbits are divided into two types, those consisting of a single point (the two eigenvalues are the same), and those diffeomorpic to the 2-sphere $S^{2}$ (the two eigenvalues are different). We shall mainly explore the behavior of $\lambda_{n}$ as functions on these spheres in the latter case.

Note that in this paper, for brevity, by eigenvalues of a boundary condition $A$ (represented by a matrix) we always mean eigenvalues of the associated boundary value problem, while eigenvalues of $A$ refer to eigenvalues of the matrix $A$.

The paper is organized as follows.

Sec. 2 is divided into two subsections. In the first subsection, we discuss the structure of $\mathrm{U}(2)$ as the space of SA boundary conditions. We identify several subsets of $U(2)$, parameterize them and show how these parameterizations are related to the ones given in (1.2) and (1.3). In the second subsection, we give a refined classification of SA boundary conditions in terms of adjoint orbits and parameterize orbits of principal type-those diffeomorphic to $S^{2}$.

Sec. 3 is devoted to briefly investigating the so-called characteristic curve $\Gamma$ which is of great importance when one considers all SA boundary conditions together. To our knowledge, this curve was first investigated in [4]. The behavior of $\Gamma$ is complicated and we hardly add any new insight into it. We only rewrite it out in our context and write down the characteristic equation in terms of it (Thm. 3.1). The advantage is that this equation is canonical

\footnotetext{
${ }^{1}$ This way of parameterizing SA extensions by $\mathrm{U}(2)$ is already known in the context of boundary triples, see for example [8, Chap. 14]
} 
and valid for all SA boundary conditions. In the end of this section, we point out the observation that $\Gamma$ has no joint point with almost all adjoint orbits of principal type. This shall imply the situation considered in Sec. 4 is general.

In Sec. 4, we investigate the behavior of $\lambda_{n}$ as a function on an adjoint orbit $\mathcal{O}$ of principal type. We show that $\lambda_{n}$ is continuous on $\mathcal{O}$. If, furthermore, $\Gamma$ has no joint point with $\mathcal{O}$, then $\lambda_{n}$ is a real analytic function on $\mathcal{O}$ and has exactly two critical points. If $\left[a_{n}, b_{n}\right]$ is the range of $\lambda_{n}$, then these $a_{n}, b_{n}, n \in \mathbb{N}$ are precisely the zeros of a certain real analytic function and $a_{n}<b_{n}<a_{n+1}<b_{n+1}$ (Thm. 4.3, 4.7, 4.9). There are two viewpoints to regard eigenvalues of S-L problems: On one side, eigenvalues are roots of the characteristic equation. On the other side, eigenvalues can also be characterized in terms of quadratic forms using the min-max principle. To obtain our results, we freely switch our viewpoint between the two if it is convenient. In the end of this section, we investigate the shape of the level subset of $\lambda$ in $\mathcal{O}$.

The last short section can be viewed as a complement of Sec. 4. We consider $\lambda_{n}$ as a function on the diagonal of the torus in $\mathrm{U}(2)$. We show that the range of $\lambda_{n}$ on $\mathrm{U}(2)$ is in fact already determined by its restriction on the diagonal (Thm. 5.1).

\section{The space of SA boundary conditions and adjoint orbits}

\subsection{The space of $S A$ boundary conditions}

Let $l_{0}$ and $l_{1}$ be the minimal and the maximal operators associated with $l$ respectively. von Neumann's abstract theory [8, Chap. 13] implies that the set $\mathcal{U}$ of all SA extensions of $l_{0}$ is parameterized by unitary transforms from $\operatorname{ker}\left(l_{1}-i \mathrm{I}\right)$ to $\operatorname{ker}\left(l_{1}+i \mathrm{I}\right)$. Since the two spaces are both 2-dimensional, topologically $\mathcal{U}$ is just $\mathrm{U}(2)$. In this description, however, there is no canonical way to identify $\mathcal{U}$ with $\mathrm{U}(2)$, because to realize such a parameterization a distinguished transform should be chosen.

Recently, an explicit and canonical way of expressing SA boundary conditions in terms of elements of $\mathrm{U}(2)$ has been found [1]. Let $y$ be a function in the Sobolev space $W_{2,2}(J)$ and 2

$$
\psi:=\left(\begin{array}{c}
y(0) \\
y(1)
\end{array}\right), \quad \dot{\psi}:=\left(\begin{array}{c}
\dot{y}(0) \\
\dot{y}(1)
\end{array}\right) .
$$

\footnotetext{
${ }^{2}$ Here we use $\dot{y}$ to denote the outward unit normal derivative of $y$. So $\dot{y}(0)=-y^{\prime}(0)$ and $\dot{y}(1)=y^{\prime}(1)$.
} 
An SA boundary condition then takes the following form:

$$
i(I+U) \dot{\psi}=(I-U) \psi
$$

where $I$ is the $2 \times 2$ identity matrix. This way we shall identify $\mathcal{U}$ with $\mathrm{U}(2)$. For the details of Eq. (2.1) and even its generalization, we refer the interested readers to [1].

Before proceeding further, we recall a description of $\mathrm{U}(2)$. Any element $g$ of $\mathrm{U}(2)$ can be decomposed into two factors:

$$
g=\sqrt{\operatorname{det} g} \cdot(g / \sqrt{\operatorname{det} g}),
$$

where $\sqrt{\operatorname{det} g} \in \mathrm{U}(1)$ is a square root of $\operatorname{det} g$, and $\frac{g}{\sqrt{\operatorname{det} g}} \in \mathrm{SU}(2)$, i.e. with determinant 1. Since there are two square roots of $\operatorname{det} g, \mathrm{U}(2)$ is the quotient of $\mathrm{U}(1) \times \mathrm{SU}(2)$ under the natural action of $\mathbb{Z}_{2}$. This result is often written as $\mathrm{U}(2)=\mathrm{U}(1) \times_{\mathbb{Z}_{2}} \mathrm{SU}(2)$. We denote the corresponding quotient map by $P$.

It's natural to classify all SA boundary conditions into two mutually exclusive subclasses according to whether $\operatorname{det}(I+U)$ equals 0 or not. Denote

$$
\mathcal{U}_{0}=\{U \in \mathcal{U} \mid \operatorname{det}(I+U)=0\}, \quad \mathcal{U}_{1}=\{U \in \mathcal{U} \mid \operatorname{det}(I+U) \neq 0\} .
$$

$\mathcal{U}_{1}$ is certainly open and dense in $\mathrm{U}(2)$. If $U \in \mathcal{U}_{1}$, then

$$
A:=-i(I+U)^{-1}(I-U)
$$

is actually a Hermitian matrix and precisely the Cayley transform of $U$. In terms of $A$, the boundary condition (2.1) can then be rewritten as

$$
\dot{\psi}=A \psi \text {. }
$$

Note that since $A$ and $U$ are in 1-1 correspondence in $\mathcal{U}_{1}, A$ can also be viewed as the coordinate in the chart $\mathcal{U}_{1} \subset \mathcal{U}$ (so topologically $\mathcal{U}_{1} \simeq \mathbb{R}^{4}$ ). Let $y_{1}, y_{2}$ be the solutions of Eq. (1.1), satisfying

$$
y_{1}(0)=1, \quad y_{1}^{\prime}(0)=0, \quad y_{2}(0)=0, \quad y_{2}^{\prime}(0)=1 \text {. }
$$

If $A=\left(\begin{array}{cc}a & b \\ \bar{b} & c\end{array}\right)$, where $a, c$ are real numbers and $b$ is complex, then the characteristic equation is

$$
\Delta(\lambda)=\operatorname{det}\left[\left(\begin{array}{cc}
0 & -1 \\
\dot{y}_{1} & \dot{y}_{2}
\end{array}\right)-\left(\begin{array}{cc}
a & b \\
\bar{b} & c
\end{array}\right)\left(\begin{array}{cc}
1 & 0 \\
y_{1} & y_{2}
\end{array}\right)\right]=0,
$$


i.e.

$$
-a \dot{y}_{2}+\left(a c-|b|^{2}\right) y_{2}-c y_{1}+\dot{y}_{1}-2 \Re b=0 .
$$

Let's come to the structure of $\mathcal{U}_{0}$. If $U \in \mathcal{U}_{0}$, we can set $U=e^{i \theta}\left(\begin{array}{cc}a & b \\ -\bar{b} & \bar{a}\end{array}\right)$, where $\theta \in[0, \pi]$, and $\left(\begin{array}{cc}a & b \\ -\bar{b} & \bar{a}\end{array}\right) \in \mathrm{SU}(2)$. Let $a=r e^{i \beta}, r \in[0,1], \beta \in[0,2 \pi)$. Then one can find that

$$
e^{i \theta}=-r \cos \beta+i \sqrt{1-r^{2} \cos ^{2} \beta} .
$$

So $U$ is completely determined by its factor in $\mathrm{SU}(2)$. But $\pm I \in \mathrm{SU}(2)$ determine the same $U=-I$. This argument shows that $\mathcal{U}_{0}$ is topologically the 3 -sphere $S^{3}$ with two points glued together ${ }^{3}$ A general element of $\mathcal{U}_{0}$ is of the following form:

$$
e^{i \theta}\left(\begin{array}{cc}
r e^{i \beta} & \sqrt{1-r^{2}} e^{i \gamma} \\
-\sqrt{1-r^{2}} e^{-i \gamma} & r e^{-i \beta}
\end{array}\right)
$$

where $\theta$ is given by Eq. (2.5) and $r \in[0,1], \beta, \gamma \in[0,2 \pi)$.

There is another interesting subset $\mathcal{U}^{\mathbb{R}} \subset \mathcal{U}$, consisting of all real SA boundary conditions. As for the shape of $\mathcal{U}^{\mathbb{R}}$ in $\mathrm{U}(2)$, we have

Proposition 2.1. $\mathcal{U}^{\mathbb{R}}=\left\{U \in \mathrm{U}(2) \mid U=U^{t}\right\}$, where the superscript t denotes the transpose of a matrix. Furthermore, with $\mathrm{U}(2)$ viewed as $\mathrm{U}(1) \times_{\mathbb{Z}_{2}} \mathrm{SU}(2)$, $\mathcal{U}^{\mathbb{R}}$ is topologically just $P\left(S^{1} \times S^{2}\right)$ (for the precise meaning, see the proof).

Proof. A real SA boundary condition is precisely one whose complex conjugate represents the same boundary condition except that $\psi, \dot{\psi}$ are replaced by $\bar{\psi}, \overline{\dot{\psi}}$. The complex conjugate of (2.1) is

$$
i(I+\bar{U}) \overline{\dot{\psi}}=-(I-\bar{U}) \bar{\psi} .
$$

It can be rewritten as

$$
i \bar{U}\left(I+\bar{U}^{-1}\right) \overline{\dot{\psi}}=\bar{U}\left(I-\bar{U}^{-1}\right) \bar{\psi}
$$

i.e.

$$
i\left(I+\bar{U}^{-1}\right) \overline{\dot{\psi}}=\left(I-\bar{U}^{-1}\right) \bar{\psi}
$$

\footnotetext{
${ }^{3}$ Topologically $\mathrm{SU}(2) \simeq S^{3}$.
} 
Then reality means $U=\bar{U}^{-1}$, which is precisely $U=U^{t}$. Let $U=e^{i \theta}\left(\begin{array}{cc}a & b \\ -\bar{b} & \bar{a}\end{array}\right)$. Then reality precisely means $b$ is purely imaginary or zero. This observation immediately leads to the conclusion that $\mathcal{U}^{\mathbb{R}}=P\left(S^{1} \times S^{2}\right)$.

Remark. In [5, Thm 3.3], there is also a description of the space of all real SA boundary conditions. However, the global picture is more clear here.

Let's now see how the boundary conditions (1.2) (1.3) look like in U(2). It is easy to see that the separated boundary conditions correspond to $U$ 's of diagonal form, forming a Cartan subgroup $\mathrm{H}$ of $\mathrm{U}(2)$, topologically a 2-torus.

For the coupled case, two subcases should be distinguished, $k_{12} \neq 0$ and $k_{12}=0$.

Proposition 2.2. If in the coupled case $k_{12} \neq 0$, then the corresponding $U$ lies in $\mathcal{U}_{1}$ and the associated Hermitian matrix is

$$
A\left(e^{i \varphi} K\right)=\frac{1}{k_{12}}\left(\begin{array}{cc}
k_{11} & -e^{-i \varphi} \\
-e^{i \varphi} & k_{22}
\end{array}\right)
$$

Proof. If $k_{12} \neq 0$, the boundary condition Eq. (1.3) can be rewritten as

$$
\dot{\psi}=\left(\begin{array}{cc}
k_{11} / k_{12} & -e^{-i \varphi} / k_{12} \\
-e^{i \varphi} / k_{12} & k_{22} / k_{12}
\end{array}\right) \psi
$$

Comparing this with Eq. (2.3), we come to the conclusion.

It is not hard to see that if $k_{12}=0$, the corresponding boundary condition cannot be rewritten as Eq. (2.3) and so the corresponding $U\left(e^{i \varphi} K\right) \in \mathcal{U}_{0}$. However, from the above proposition, we can obtain a unified expression of $U\left(e^{i \varphi} K\right)$ no matter whether $k_{12}=0$ or not:

Proposition 2.3. For the coupled boundary condition (1.3), the corresponding element $U\left(e^{i \varphi} K\right) \in \mathrm{U}(2)$ is

$\frac{1}{k_{12}-k_{21}+i\left(k_{11}+k_{22}\right)}\left(\begin{array}{cc}k_{12}+k_{21}+i\left(k_{22}-k_{11}\right) & 2 i e^{-i \varphi} \\ 2 i e^{i \varphi} & k_{12}+k_{21}-i\left(k_{22}-k_{11}\right)\end{array}\right)$

and it has -1 as its eigenvalue if and only if $k_{12}=0$. 
Proof. If $k_{12} \neq 0$, then from Prop. 2.2, $U\left(e^{i \varphi} K\right) \in \mathcal{U}_{1}$ and

$$
U\left(e^{i \varphi} K\right)=\left[I-i A\left(e^{i \varphi} K\right)\right]\left[I+i A\left(e^{i \varphi} K\right)\right]^{-1}
$$

due to Eq. (2.2). This leads to the expression as required. Obviously this expression extends smoothly to the case $k_{12}=0$.

That $\operatorname{det}\left[I+U\left(e^{i \varphi} K\right)\right]=0$ is equivalent to

$$
k_{12}\left[k_{12}-k_{21}+i\left(k_{22}+k_{11}\right)\right]=0
$$

which holds if and only if $k_{12}=0$.

For the case $k_{12}=0$, we also have

Proposition 2.4. If in the coupled case $k_{12}=0$, then in terms of $r, \beta, \gamma$, the matrix $e^{i \varphi} K$ is determined by (without loss of generality, we set $k_{11}>0$ )

$$
\begin{gathered}
k_{11}=\frac{\sqrt{1-r^{2} \cos ^{2} \beta}+r \sin \beta}{\sqrt{1-r^{2}}} \\
k_{21}=\frac{-2 r \cos \beta}{\sqrt{1-r^{2}}} \\
e^{i \varphi}=e^{-i\left(\gamma+\frac{\pi}{2}\right)}
\end{gathered}
$$

Proof. In terms of $r, \beta, \gamma$, the associated boundary condition is

$$
\left\{\begin{array}{l}
y(1)=\frac{\sqrt{1-r^{2} \cos ^{2} \beta}+r \sin \beta}{\sqrt{1-r^{2}}} e^{-i\left(\gamma+\frac{\pi}{2}\right)} y(0), \\
y^{\prime}(1)=-\frac{2 r \cos \beta}{\sqrt{1-r^{2}}} e^{-i\left(\gamma+\frac{\pi}{2}\right)} y(0)+\frac{\sqrt{1-r^{2} \cos ^{2} \beta}-r \sin \beta}{\sqrt{1-r^{2}}} e^{-i\left(\gamma+\frac{\pi}{2}\right)} y^{\prime}(0) .
\end{array}\right.
$$

Comparing this with Eq. (1.3), we get the conclusion.

$\mathcal{U}_{0}$ has been investigated in other way in the literature. From the above discussion, it is easy to see that $\mathcal{U}_{0}$ is actually the set $\mathscr{J}^{\mathbb{C}}$ in [4].

Remark. No matter whether $k_{12}=0$ or not, the eigenvalues of $U\left(e^{i \varphi} K\right)$ are independent of $\varphi$. So for a fixed $K, U\left(e^{i \varphi} K\right), \varphi \in[0,2 \pi)$ all lie in the same adjoint orbit, tracing out a circle. There is a beautiful inequality among eigenvalues of S-L problems when the boundary condition varies only on this circle [2]. 


\subsection{Adjoint orbits}

Let $\mathrm{H} \subset \mathrm{U}(2)$ be the Cartan subgroup as in the last subsection, and $W\left(\cong \mathbb{Z}_{2}\right)$ the corresponding Weyl group. Then the quotient $\mathrm{H} / W$ is a 2 dimensional manifold with boundary-in fact, it is topologically the famous Mobius strip. $\mathrm{H} / W$ can be viewed as the space of adjoint orbits in $\mathrm{U}(2)$, with each interior point representing an adjoint orbit of principal type and with each point on the boundary representing an adjoint orbit consisting of a single matrix. In this sense, a generic adjoint orbit is diffeomorphic to $S^{2}$. Let $\Pi: \mathrm{U}(2) \rightarrow \mathrm{H} / W$ be the quotient map. We refer the reader to [9] for the basics of compact Lie group.

Since both $\mathcal{U}_{0}$ and $\mathcal{U}_{1}$ are invariant under the adjoint action, an adjoint orbit would lie entirely either in $\mathcal{U}_{0}$ or $\mathcal{U}_{1}$. This, of course, leads to a more refined classification of SA boundary conditions-each adjoint orbit represents a subclass. In this subsection, we mainly consider orbits of principal type. These are in fact real analytic 2-dimensional manifolds.

By (2.2), $\mathcal{U}_{1}$ is diffeomorphic to the space $\mathcal{M}$ of $2 \times 2$ Hermitian matrices and, the adjoint action of $\mathrm{U}(2)$ on $\mathcal{U}_{1}$ corresponds to the one on $\mathcal{M}$. This way, we can identify adjoint orbits in $\mathcal{U}_{1}$ with adjoint orbits in $\mathcal{M}$. An adjoint orbit $\mathcal{O} \subset \mathcal{M}$ is characterized by its eigenvalues $\zeta_{1}>\zeta_{2}$. Let $\mu=\frac{\zeta_{1}+\zeta_{2}}{2}$, $\nu=\frac{\zeta_{1}-\zeta_{2}}{2}$ and denote the adjoint orbit by $\mathcal{O}_{\mu, \nu}$.

Proposition 2.5. A general element in $\mathcal{O}_{\mu, \nu}$ is of the following form:

$$
A=\left(\begin{array}{cc}
\mu-\nu \cos 2 \theta & \nu \sin 2 \theta \cdot e^{-i \gamma} \\
\nu \sin 2 \theta \cdot e^{i \gamma} & \mu+\nu \cos 2 \theta
\end{array}\right), \quad \gamma \in[0,2 \pi), \quad \theta \in\left[0, \frac{\pi}{2}\right] .
$$

Proof. $\mathcal{O}_{\mu, \nu}$ is the adjoint orbit through $\left(\begin{array}{cc}\mu-\nu & 0 \\ 0 & \mu+\nu\end{array}\right)$. Then each element in $\mathcal{O}_{\mu, \nu}$ can be represented by

$$
\left(\begin{array}{cc}
a & b \\
-\bar{b} & \bar{a}
\end{array}\right)\left(\begin{array}{cc}
\mu-\nu & 0 \\
0 & \mu+\nu
\end{array}\right)\left(\begin{array}{cc}
a & b \\
-\bar{b} & \bar{a}
\end{array}\right)^{-1}
$$

for some $\left(\begin{array}{cc}a & b \\ -\bar{b} & \bar{a}\end{array}\right) \in \mathrm{SU}(2)$. We can even further require $a \geq 0$. Setting $a=\cos \theta, \theta \in\left[0, \frac{\pi}{2}\right]$, and $b=\sin \theta \cdot e^{-i \gamma}$ then leads to the representation.

Remark. From Prop. 2.2, we can see that $\gamma$ essentially contains the same geometric content as $\varphi$ in Eq. (1.3). Note that $\theta=0, \frac{\pi}{2}$ actually correspond 
to the only two separated boundary conditions in $\mathcal{O}_{\mu, \nu}$. It is easy to find that in $\mathcal{O}_{\mu, \nu}$, real boundary conditions lie precisely on the circle formed by the two semi-circles $\gamma=0$ and $\gamma=\pi$. It will soon be clear that this is a general property of orbits of principal type.

An adjoint orbit in $\mathcal{U}_{0}$ is determined by the other eigenvalue $e^{i \alpha}$ ( $\alpha \in$ $[0, \pi) \cup(\pi, 2 \pi))$ besides -1 . We shall denote the orbit by $\mathcal{O}_{\alpha}$. Since in this case,

$$
\operatorname{tr} U=e^{i \theta}(a+\bar{a})=-1+e^{i \alpha},
$$

we find $\Re a=\sin \frac{\alpha}{2}$ if $\alpha \in[0, \pi)$ and, $\Re a=-\sin \frac{\alpha}{2}$ if $\alpha \in(\pi, 2 \pi)$.

Proposition 2.6. For $\alpha \in[0, \pi)$, a general element of $\mathcal{O}_{\alpha}$ is of the following form:

$$
U=i e^{i \alpha / 2}\left(\begin{array}{cc}
\sin \frac{\alpha}{2}+i t & \sqrt{\cos ^{2} \frac{\alpha}{2}-t^{2}} e^{i \gamma} \\
-\sqrt{\cos ^{2} \frac{\alpha}{2}-t^{2}} e^{-i \gamma} & \sin \frac{\alpha}{2}-i t
\end{array}\right),
$$

where $t \in\left[-\cos \frac{\alpha}{2}, \cos \frac{\alpha}{2}\right]$ and $\gamma \in[0,2 \pi)$.

For $\alpha \in(\pi, 2 \pi)$, a general element of $\mathcal{O}_{\alpha}$ is of the following form:

$$
U=-i e^{i \alpha / 2}\left(\begin{array}{cc}
-\sin \frac{\alpha}{2}+i t & \sqrt{\cos ^{2} \frac{\alpha}{2}-t^{2}} e^{i \gamma} \\
-\sqrt{\cos ^{2} \frac{\alpha}{2}-t^{2}} e^{-i \gamma} & -\sin \frac{\alpha}{2}-i t
\end{array}\right)
$$

where $t \in\left[\cos \frac{\alpha}{2},-\cos \frac{\alpha}{2}\right]$ and $\gamma \in[0,2 \pi)$.

Proof. By Eq. (2.5),

$$
e^{i \theta}=-\sin \frac{\alpha}{2}+i \cos \frac{\alpha}{2}=i e^{i \alpha / 2}
$$

if $\alpha \in[0, \pi)$, and

$$
e^{i \theta}=\sin \frac{\alpha}{2}-i \cos \frac{\alpha}{2}=-i e^{i \alpha / 2}
$$

if $\alpha \in(\pi, 2 \pi)$. The conclusion then easily follows.

\section{The characteristic curve}

The characteristic curve $\Gamma: \mathbb{R} \rightarrow \mathrm{U}(2)$ is a parameterized curve, the image of which consists of all SA boundary conditions having a double eigenvalue. This curve contains all information concerning eigenvalues of SA boundary conditions (of course, if one puts eigenfunctions aside) [5]. 
From (2.1), it is easy to find that $\Gamma$ is of the following form :

$$
\Gamma(\lambda)=\frac{1}{y_{2}-\dot{y}_{1}+i \dot{y}_{2}+i y_{1}}\left(\begin{array}{cc}
y_{2}+\dot{y}_{1}+i \dot{y}_{2}-i y_{1} & 2 i \\
2 i & y_{2}+\dot{y}_{1}-i \dot{y}_{2}+i y_{1}
\end{array}\right),
$$

where $\lambda \in \mathbb{R}$. The image of $\Gamma$ is completely included in $\mathcal{U}^{\mathbb{R}}$. $\Pi \circ \Gamma$ is a curve in $\mathrm{H} / W$. We call $\Pi \circ \Gamma$ the induced curve of $\Gamma$. $\Pi \circ \Gamma$ is characterized by the two eigenvalues of $\Gamma(\lambda)$, say,

$$
\kappa_{ \pm}(\lambda)=\frac{y_{2}+\dot{y}_{1} \pm i \sqrt{4+\left(\dot{y}_{2}-y_{1}\right)^{2}}}{y_{2}-\dot{y}_{1}+i \dot{y}_{2}+i y_{1}} .
$$

Proposition 3.1. In terms of $\Gamma(\lambda)$, the characteristic equation for an $S A$ boundary condition $U$ can be written in the following form:

$$
\operatorname{det}(U-\Gamma(\lambda))=0 .
$$

The subset $S_{\lambda} \subset \mathcal{U}$ of boundary conditions with $\lambda$ as an eigenvalue is diffeomorphic to $\mathcal{U}_{0}$.

Proof. U(2) acts on itself by left translation and $S_{\lambda}$ can be represented as $-\Gamma(\lambda) \mathcal{U}_{0}$. By Eq. (3.1), $S_{\lambda}$ is diffeomorphic to $\mathcal{U}_{0}$, i.e. a 3 -sphere with 2 points glued together. This observation was already noted in [5], but in a more complicated language.

Corollary 3.2. The matrix $\Gamma(\lambda)$ has -1 as an eigenvalue if and only if $\lambda=\lambda_{n}^{D}$ for some $n \in \mathbb{N}$. Therefore, the characteristic curve $\Gamma$ intersects $\mathcal{U}_{0}$ countably infinite times.

Proof. This is obvious.

Remark. However, the above result doesn't mean that $\Gamma$ has infinitely many intersection points with $\mathcal{U}_{0}$. Besides, -1 can be replaced by $e^{i \theta} I, \theta \in[0,2 \pi)$, and similar result holds.

Example 3.3. Let $q \equiv 0$. Then for $\lambda>0, y_{1}(x, \lambda)=\cos \sqrt{\lambda} x, y_{2}(x, \lambda)=$ $\frac{\sin \sqrt{\lambda} x}{\sqrt{\lambda}}$. The two eigenvalues of $\Gamma(\lambda)$ are

$$
\kappa_{ \pm}(\lambda)=\frac{\left(\frac{1}{\sqrt{\lambda}}-\sqrt{\lambda}\right) \sin \sqrt{\lambda} \pm 2 i}{\left(\frac{1}{\sqrt{\lambda}}+\sqrt{\lambda}\right) \sin \sqrt{\lambda}+2 i \cos \sqrt{\lambda}} .
$$


For the Dirichlet boundary condition, $\lambda_{n}^{D}=(n+1)^{2} \pi^{2}, \kappa_{ \pm}\left(\lambda_{n}^{D}\right)= \pm(-1)^{n+1}$. So in this case, the intersection points of $\Gamma$ and $\mathcal{U}_{0}$ all lie in the orbit through $\left(\begin{array}{cc}1 & 0 \\ 0 & -1\end{array}\right)$. In fact, there are only two such points, i.e. $\pm\left(\begin{array}{ll}0 & 1 \\ 1 & 0\end{array}\right)$.

Since the space $\mathrm{H} / W$ parameterizing all adjoint orbits is of dimension 2 , and the induced curve of $\Gamma$ is analytic and, of course, of dimension 1 , the characteristic curve $\Gamma$ would not go through a generic adjoint orbit of principal type.

\section{4. $\lambda_{n}$ as functions on adjoint orbits of principal type}

In this section, by adjoint orbits we will always refer to those of principal type. We mainly consider adjoint orbits which have no joint point with the characteristic curve $\Gamma$. From the last section, we know a generic adjoint orbit is of this kind. By $\lambda_{n}^{N}$, we denote the $n$-th eigenvalue of the Neumann boundary condition.

For the orbit $\mathcal{O}_{\mu, \nu}$, by Eq. (2.4) the corresponding characteristic equation is

$$
(\mu-\nu \cos 2 \theta) \dot{y}_{2}+\left(\nu^{2}-\mu^{2}\right) y_{2}+(\mu+\nu \cos 2 \theta) y_{1}-\dot{y}_{1}=-2 \nu \sin 2 \theta \cdot \cos \gamma .
$$

Lemma 4.1. Let $\lambda_{n}^{ \pm}$be the $n$-th eigenvalues of the boundary conditions

$$
\dot{y}(0)=(\mu-\nu) y(0), \quad \dot{y}(1)=(\mu-\nu) y(1)
$$

and

$$
\dot{y}(0)=(\mu+\nu) y(0), \quad \dot{y}(1)=(\mu+\nu) y(1)
$$

respectively. Then the function $\lambda_{n}$ on $\mathcal{O}_{\mu, \nu}$ satisfies

$$
\lambda_{n}^{-} \leq \lambda_{n} \leq \lambda_{n}^{+}
$$

In particular, by the continuity principle, $\lambda_{n}$ is continuous on $\mathcal{O}_{\mu, \nu}$.

Proof. For $A \in \mathcal{O}_{\mu, \nu}$, the associated quadratic form is

$$
Q(y)=\int_{0}^{1}\left|y^{\prime}\right|^{2} d x+\int_{0}^{1} q(x)|y|^{2} d x-\psi^{\dagger} A \psi, \quad y \in H^{1},
$$

where $H^{1}$ is the Sobolev space $W_{1,2}(J)$. 
Note that

$$
\psi^{\dagger} A \psi=\mu|\psi|^{2}+\left(|y(1)|^{2}-|y(0)|^{2}\right) \nu \cos 2 \theta+2 \nu \Re\left[\bar{y}(0) y(1) e^{-i \gamma}\right] \sin 2 \theta .
$$

By the inequality $2|a b| \leq|a|^{2}+|b|^{2}$, we have

$$
2 \Re\left[\bar{y}(0) y(1) e^{-i \gamma}\right] \sin 2 \theta \leq(1+\cos 2 \theta)|y(0)|^{2}+(1-\cos 2 \theta)|y(1)|^{2}
$$

and

$$
2 \Re\left[\bar{y}(0) y(1) e^{-i \gamma}\right] \sin 2 \theta \geq-(1-\cos 2 \theta)|y(0)|^{2}-(1+\cos 2 \theta)|y(1)|^{2} .
$$

Therefore, we come to the estimation

$$
(\mu-\nu)|\psi|^{2} \leq \psi^{\dagger} A \psi \leq(\mu+\nu)|\psi|^{2} .
$$

The conclusion then follows from the variational characterization of $\lambda_{n}(A)-$ the min-max principle.

Remark. The boundedness from below of $\lambda_{n}$ on $\mathcal{O}_{\mu, \nu}$ is actually a conclusion of [4] that $\lambda_{n}$ is continuous on $\mathcal{U}_{1}$, together with the fact that $S^{2}$ is compact. Conversely, minor modification of the proof of Lemma 4.1 gives another proof of that $\lambda_{n}$ is continuous on $\mathcal{U}_{1}$.

Proposition 4.2. $\lambda_{n}$ is a continuous function on $\mathcal{U}_{1}$.

Proof. For any given $A_{0} \in \mathcal{U}_{1}$, let $\mathcal{O}_{\mu_{0}, \nu_{0}}$ be the orbit through $A_{0}$ (we allow $\nu_{0}$ to be 0 here). Then for $\delta>0$, the set

$$
V_{\delta}=\cup_{\mu+\nu<\mu_{0}+\nu_{0}+\delta} \mathcal{O}_{\mu, \nu}
$$

is an open neighbourhood of $A_{0}$. Note that for $A \in V_{\delta}$,

$$
\left(\mu_{0}+\nu_{0}+\delta\right)|\psi|^{2} \geq \psi^{\dagger} A \psi
$$

The min-max principle implies that $\lambda_{0}$ is bounded from below on $V_{\delta}$ and thus $\lambda_{n}$ is continuous on $V_{\delta}$, and in particular, continuous at $A_{0}$.

Theorem 4.3. Assume that $\mathcal{O}_{\mu, \nu}$ has no joint point with $\Gamma$. Then for each $n$, $\lambda_{n}$ as a function on $\mathcal{O}_{\mu, \nu}$ is real analytic, and has exactly two critical points. Let $\left[a_{n}, b_{n}\right]$ be the range of $\lambda_{n}$ on $\mathcal{O}_{\mu, \nu}$. Then for each $n$,

$$
a_{n}<b_{n}<a_{n+1}<b_{n+1} .
$$

These $a_{n}, b_{n}, n=0,1,2, \cdots$ are exactly roots of the following equation:

$$
\nu^{2}\left(\dot{y}_{2}-y_{1}\right)^{2}+4 \nu^{2}=\left[\mu\left(\dot{y}_{2}+y_{1}\right)+\left(\nu^{2}-\mu^{2}\right) y_{2}-\dot{y}_{1}\right]^{2} .
$$


Proof. Denote the LHS of Eq. (4.1) by $D(\lambda, p)$, viewed as a function on $\mathbb{R} \times \mathcal{O}_{\mu, \nu}$. Since $\mathcal{O}_{\mu, \nu}$ has no joint point with $\Gamma,\left.\frac{\partial D}{\partial \lambda}\right|_{\lambda_{n}(p), p} \neq 0$ for any $p \in \mathcal{O}_{\mu, \nu}$. Besides, $D(\lambda, p)$ and the RHS of Eq. (4.1) are real analytic functions on $\mathbb{R} \times \mathcal{O}_{\mu, \nu}$. So by the implicit function theorem, $\lambda_{n}$ is a real analytic function on $\mathcal{O}_{\mu, \nu}$.

It is easy to find that for a critical point $p$, we must have $\sin \gamma=0$. This implies that all critical points must lie on the circle $C_{0}$ formed by the two semi-circles $\gamma=0$ and $\gamma=\pi$. So, to find all critical points of $\lambda_{n}$ on $\mathcal{O}_{\mu, \nu}$, we only need to find all critical points of $\lambda_{n}$ on $C_{0}$. Now consider the characteristic equation restricted on $C_{0}$, i.e.

$$
\nu\left(\dot{y}_{2}-y_{1}\right) \cos 2 \theta-2 \nu \sin 2 \theta=\mu\left(\dot{y}_{2}+y_{1}\right)+\left(\nu^{2}-\mu^{2}\right) y_{2}-\dot{y}_{1},
$$

where $\theta \in\left(-\frac{\pi}{2}, \frac{\pi}{2}\right]$. For a given $\lambda \in \mathbb{R}$, there are at most two values of $\theta$ satisfying the above equation. It is an element calculation to show that $\lambda_{n}$ has no degenerate critical point. These together imply that there are at most two critical points of $\lambda_{n}$ as a function on $C_{0}$. Since $C_{0}$ is compact, we know that there are precisely two critical points, one the maximizer and the other the minimizer.

Any critical value $\kappa$ of $\lambda_{n}$ must satisfy Eq. (4.2). Conversely, it is not hard to find that any root $\kappa$ of Eq. (4.2) must be a critical value of some $\lambda_{n}$. By the uniqueness of minimizer and maximizer, $\kappa=a_{n}$ or $b_{n}$.

If $a_{n+1}=\lambda_{n+1}\left(p_{0}\right)$ for some $p_{0} \in C_{0}$, then

$$
a_{n+1}>\lambda_{n}\left(p_{0}\right) \geq a_{n} .
$$

We only need to check that $a_{n+1}>b_{n}$. If it is not the case, then $a_{n+1} \in$ $\left(a_{n}, b_{n}\right]$ and there is another point $p_{1} \in C_{0}$ such that

$$
\lambda_{n}\left(p_{1}\right)=\lambda_{n+1}\left(p_{0}\right)=a_{n+1} .
$$

If $p_{1}=p_{0}$, this means that $a_{n+1}$ is a double eigenvalue of the boundary condition $p_{0}$, contradicting that $\Gamma$ has no joint point with $\mathcal{O}_{\mu, \nu}$; if $p_{1} \neq p_{0}$, then for $\lambda=a_{n+1}$, Eq. (4.3) of $\theta$ has at least two different solutions. This contradicts the fact that $a_{n+1}$ is the unique minimum of $\lambda_{n+1}$. The proof is then completed.

Example 4.4. Let $q \equiv 0$. Then for $\lambda>0$ Eq. (4.3) is

$$
-2 \nu \sin 2 \theta=2 \mu \cos \sqrt{\lambda}+\left(\nu^{2}-\mu^{2}\right) \frac{\sin \sqrt{\lambda}}{\sqrt{\lambda}}+\sqrt{\lambda} \cos \sqrt{\lambda}
$$


$\theta= \pm \frac{\pi}{4}$ are the common critical points of all $\lambda_{n}$ such that $\lambda_{n}>0$. Eq. (4.2) now is

$$
2 \mu \cos \sqrt{\lambda}+\left(\nu^{2}-\mu^{2}\right) \frac{\sin \sqrt{\lambda}}{\sqrt{\lambda}}+\sqrt{\lambda} \cos \sqrt{\lambda}= \pm 2 \nu .
$$

If $\mu=\nu$, then the above equation obtains a more accessible form:

$$
\cos \sqrt{\lambda}= \pm \frac{2 \nu}{2 \nu+\sqrt{\lambda}}
$$

From [4, Thm. 3.73], we can derive that, nearly all points in $\mathcal{U}_{0}$ are discontinuity points of $\lambda_{n}$ as a function on $\mathrm{U}(2)$. This, of course, doesn't exclude the possibility that $\lambda_{n}$ is continuous on adjoint orbits lying in $\mathcal{U}_{0}$.

For the orbit $\mathcal{O}_{\alpha}$ with $\alpha \in[0, \pi)$, the associated characteristic equation is

$$
\left(-\cos \frac{\alpha}{2}+t\right) \dot{y}_{2}+\left(-\cos \frac{\alpha}{2}-t\right) y_{1}-2 \sin \frac{\alpha}{2} y_{2}=2 \cos \left(\gamma+\frac{\pi}{2}\right) \sqrt{\cos ^{2} \frac{\alpha}{2}-t^{2}}
$$

Lemma 4.5. On the orbit $\mathcal{O}_{\alpha}$ with $\alpha \in[0, \pi)$,

$$
\lambda_{n} \geq \lambda_{n}^{N}, \quad n=0,1,2, \cdots
$$

In particular, by the continuity principle, $\lambda_{n}$ is continuous on $\mathcal{O}_{\alpha}$.

Proof. If $t \neq \cos \frac{\alpha}{2}$, the associated quadratic form is

$$
Q_{1}(y)=\int_{0}^{1}\left|y^{\prime}\right|^{2} d x+\int_{0}^{1} q(x)|y|^{2} d x+\frac{2 \sin \frac{\alpha}{2}}{\cos \frac{\alpha}{2}-t}|y(0)|^{2}, y \in H_{\gamma, t}^{1},
$$

where

$$
H_{\gamma, t}^{1}=\left\{y \in H^{1} \mid y(1)=e^{-i\left(\gamma+\frac{\pi}{2}\right)} \sqrt{\frac{\cos \frac{\alpha}{2}+t}{\cos \frac{\alpha}{2}-t}} y(0)\right\} \subset H^{1} .
$$

Note that in this case, by the min-max principle,

$$
\lambda_{n}=\min _{S_{n+1} \subset H_{\gamma, t}^{1},} \max _{y \in S_{n+1}-\{0\}} \frac{Q_{1}(y)}{\|y\|^{2}},
$$

where $S_{n+1}$ ranges over all $n+1$-dimensional subspaces of $H_{\gamma, t}^{1}$. Since

$$
\min _{S_{n+1} \subset H_{\gamma, t}^{1}} \max _{y \in S_{n+1}-\{0\}} \frac{Q_{1}(y)}{\|y\|^{2}} \geq \min _{S_{n+1} \subset H_{\gamma, t}^{1}} \max _{y \in S_{n+1}-\{0\}} \frac{Q_{0}(y)}{\|y\|^{2}}
$$


where $Q_{0}(y)=\int_{0}^{1}\left|y^{\prime}\right|^{2} d x+\int_{0}^{1} q(x)|y|^{2} d x$, and

$$
\lambda_{n}^{N}=\min _{S_{n+1} \subset H^{1}} \max _{y \in S_{n+1}-\{0\}} \frac{Q_{0}(y)}{\|y\|^{2}}
$$

where $S_{n+1}$ ranges over all $n+1$-dimensional subspaces of $H^{1}$, we must have

$$
\lambda_{n} \geq \lambda_{n}^{N}
$$

If $t=\cos \frac{\alpha}{2}$, the associated quadratic form is

$$
Q_{2}(y)=\int_{0}^{1}\left|y^{\prime}\right|^{2} d x+\int_{0}^{1} q(x)|y|^{2} d x+\tan \frac{\alpha}{2}|y(1)|^{2}, \quad y \in H^{1}, y(0)=0 .
$$

A similar argument then leads to the inequality $\lambda_{n} \geq \lambda_{n}^{N}$.

Corollary 4.6. Let $\Omega:=\cup_{\alpha \in[0, \pi)} \mathcal{O}_{\alpha}$. Then $\lambda_{n}$ are continuous functions on $\Omega$.

Proof. Note that $\lambda_{0}^{N}$ is independent of the orbit parameter $\alpha$. The inequality in Lemma 4.5 holds uniformly on $\Omega$. By the continuity principle, the conclusion follows.

Theorem 4.7. Assume that $\mathcal{O}_{\alpha}$ with $\alpha \in[0, \pi)$ has no joint point with $\Gamma$. Then on $\mathcal{O}_{\alpha}, \lambda_{n}$ is real analytic, and has exactly two critical points. Let $\left[a_{n}, b_{n}\right]$ be the range of $\lambda_{n}$ on $\mathcal{O}_{\alpha}$. Then for each $n$,

$$
a_{n}<b_{n}<a_{n+1}<b_{n+1} .
$$

These $a_{n}, b_{n}, n=0,1,2, \cdots$ are exactly roots of the following equation:

$$
\left(y_{1}-\dot{y}_{2}\right)^{2}+4=\left(\dot{y}_{2}+y_{1}+2 y_{2} \tan \frac{\alpha}{2}\right)^{2} .
$$

Proof. Let $t=\cos \frac{\alpha}{2} \sin \tau, \tau \in\left[-\frac{\pi}{2}, \frac{\pi}{2}\right]$. Then the characteristic equation becomes

$$
2 \cos \left(\gamma+\frac{\pi}{2}\right) \cos \tau+(1-\sin \tau) \dot{y}_{2}+(1+\sin \tau) y_{1}+2 y_{2} \tan \frac{\alpha}{2}=0 .
$$

Then the argument in the proof of Thm. 4.3 still holds. We omit the details here. 
For the orbit $\mathcal{O}_{\alpha}$ with $\alpha \in(\pi, 2 \pi)$, the corresponding characteristic equation is

$$
\left(\cos \frac{\alpha}{2}+t\right) \dot{y}_{2}+\left(\cos \frac{\alpha}{2}-t\right) y_{1}+2 \sin \frac{\alpha}{2} y_{2}=2 \cos \left(\gamma+\frac{\pi}{2}\right) \sqrt{\cos ^{2} \frac{\alpha}{2}-t^{2}}
$$

Lemma 4.8. On the orbit $\mathcal{O}_{\alpha}$ with $\alpha \in(\pi, 2 \pi), \lambda_{0}$ is bounded from below. In particular, by the continuity principle, $\lambda_{n}$ is continuous on $\mathcal{O}_{\alpha}$.

Proof. We only need to prove that for sufficiently negative $\lambda$, Eq. (4.5) cannot hold for any $\gamma$ and $t$. For this purpose, we should use the following estimations for sufficiently negative $\lambda=-s^{2}(s>0)$ :

$y_{1}(1, \lambda)=\cosh s+O\left(\frac{e^{s}}{s}\right), \quad y_{2}(1, \lambda)=\frac{\sinh s}{s}+O\left(\frac{e^{s}}{s^{2}}\right), \quad \dot{y}_{2}(1, \lambda)=\cosh s+O\left(\frac{e^{s}}{s}\right)$.

These results are not hard to obtain from Lemma 2.1.1 and Lemma 2.1.2 of [6, Chap. 2]. Note that unlike in the previous situation, the continuity of $q$ is used to obtain these estimations.

Divide the LHS of Eq. (4.5) by $\cosh s$. Then for sufficiently large $s$, the result $<\cos \frac{\alpha}{2}$. Divide the RHS of Eq. (4.5) by $\cosh s$. Then for sufficiently $\operatorname{large} s$, the result $>\cos \frac{\alpha}{2}$. This is exactly what we want.

Remark. For $t<-\cos \frac{\alpha}{2}$, the associated quadratic form is

$$
Q_{1}(y)=\int_{0}^{1}\left|y^{\prime}\right|^{2} d x+\int_{0}^{1} q(x)|y|^{2} d x+\frac{2 \sin \frac{\alpha}{2}}{\cos \frac{\alpha}{2}+t}|y(0)|^{2}, y \in H_{\gamma, t}^{1},
$$

where

$$
H_{\gamma, t}^{1}=\left\{y \in H^{1} \mid y(1)=e^{-i\left(\gamma+\frac{\pi}{2}\right)} \sqrt{\frac{-\cos \frac{\alpha}{2}+t}{-\cos \frac{\alpha}{2}-t}} y(0)\right\} \subset H^{1} .
$$

The argument in the proof of Lemma 4.5 fails to hold. The situation is similar for $t=-\cos \frac{\alpha}{2}$. This is the reason why we have turned to the several estimations in the proof of the above lemma.

Theorem 4.9. Assume that $\mathcal{O}_{\alpha}$ with $\alpha \in(\pi, 2 \pi)$ has no joint point with $\Gamma$. Then on $\mathcal{O}_{\alpha}, \lambda_{n}$ is real analytic, and has exactly two critical points. Let $\left[a_{n}, b_{n}\right]$ be the range of $\lambda_{n}$ on $\mathcal{O}_{\alpha}$. Then for each $n$,

$$
a_{n}<b_{n}<a_{n+1}<b_{n+1} .
$$


These $a_{n}, b_{n}, n=0,1,2, \cdots$ are exactly roots of the following equation:

$$
\left(y_{1}-\dot{y}_{2}\right)^{2}+4=\left(\dot{y}_{2}+y_{1}+2 y_{2} \tan \frac{\alpha}{2}\right)^{2} .
$$

Proof. The proof is similar to that of Thm. 4.7 and we omit the details.

Remark. In [2], the authors obtained a general inequality among eigenvalues of different coupled boundary conditions. In fact, these boundary conditions lie on the circle parameterized by $\gamma$ in our adjoint orbit $\mathcal{O}$. In [3], this inequality was re-derived via variational characterization of eigenvalues. To certain extent, our inequality $a_{n}<b_{n}<a_{n+1}<b_{n+1}$ can be viewed as an extension in this direction-we consider an adjoint orbit rather than a circle in it.

Example 4.10. Let $q \equiv 0$. Then for $\lambda>0$, the equation in Thm. 4.7 or Thm. 4.9 is

$$
\cos \sqrt{\lambda}+\frac{\sin \sqrt{\lambda}}{\sqrt{\lambda}} \tan \frac{\alpha}{2}= \pm 1 .
$$

The critical points are $t=0$ and $\gamma=0$ or $\pi$.

As a conclusion of this section, we shall find out the level set $\Lambda^{\kappa}$ in an adjoint orbit $\mathcal{O}$ consisting of boundary conditions with $\kappa$ as an eigenvalue.

Theorem 4.11. Let $\mathcal{O}$ be an adjoint orbit and $p \in \mathcal{O}$. If $\lambda_{n}(p)=\kappa$ for some $n$, then the level set $\Lambda^{\kappa}$ is a set either consisting of a single point or diffeomorphic to a circle.

Proof. Let $\zeta_{1} \neq \zeta_{2}$ be the two eigenvalues of $\mathcal{O} \subset \mathrm{U}(2)$ and $\varrho_{1} \neq \varrho_{2}$ the two eigenvalues of $\Gamma(\kappa)$. The general element of $\mathcal{O}$ is of the following form:

$$
U(x, \gamma)=\left(\begin{array}{cc}
\zeta_{1} x+\zeta_{2}(1-x) & \left(\zeta_{2}-\zeta_{1}\right) \sqrt{x(1-x)} e^{i \gamma} \\
\left(\zeta_{2}-\zeta_{1}\right) \sqrt{x(1-x)} e^{-i \gamma} & \zeta_{1}(1-x)+\zeta_{2} x
\end{array}\right),
$$

where $x \in[0,1], \gamma \in[0,2 \pi) . \quad \Lambda^{\kappa} \subset \mathcal{O}$ is characterized by the equation $\operatorname{det}(U(x, \gamma)-\Gamma(\kappa))=0$. Since we are only interested in the shape of $\Lambda^{\kappa}$, we can safely set $\Gamma(\kappa)=\left(\begin{array}{cc}\varrho_{1} & 0 \\ 0 & \varrho_{2}\end{array}\right)$. This implies the following:

$$
x=-\frac{\left(\zeta_{2}-\varrho_{1}\right)\left(\zeta_{1}-\varrho_{2}\right)}{\left(\varrho_{1}-\varrho_{2}\right)\left(\zeta_{2}-\zeta_{1}\right)} .
$$




$$
1-x=\frac{\left(\zeta_{1}-\varrho_{1}\right)\left(\zeta_{2}-\varrho_{2}\right)}{\left(\varrho_{1}-\varrho_{2}\right)\left(\zeta_{2}-\zeta_{1}\right)},
$$

If at least one of $\zeta_{1}, \zeta_{2}$ coincides with $\varrho_{1}$ or $\varrho_{2}$, then $x$ or $1-x$ equals zero and $\Lambda^{\kappa}$ consists of the only point $p=\left(\begin{array}{cc}\zeta_{1} & 0 \\ 0 & \zeta_{2}\end{array}\right)$ or $\left(\begin{array}{cc}\zeta_{2} & 0 \\ 0 & \zeta_{1}\end{array}\right)$.

If $\zeta_{1}, \zeta_{2}$ are both different from $\varrho_{1}$ and $\varrho_{2}$, then both $x$ and $1-x$ are nonzero and determined by these values. It follows that $\Lambda^{\kappa}$ is diffeomorphic to $S^{1}$, parameterized by $\gamma$.

Remark. If $\mathcal{O}$ has no joint point with $\Gamma$, then from our previous result, $\Lambda^{\kappa}$ is actually the level- $\kappa$ set of $\lambda_{n}$. If $\kappa=a_{n}$ or $b_{n}$, then $\Lambda^{\kappa}$ consists of the minimizer or maximizer of $\lambda_{n}$. For other values of $\kappa, \Lambda^{\kappa}$ are all diffeomorphic to $S^{1}$.

\section{5. $\lambda_{n}$ as functions on the boundary circle of $\mathrm{H} / W$}

In the previous sections, we have mainly analyzed the behavior of $\lambda_{n}$ as functions on generic adjoint orbits represented by interior points in $\mathrm{H} / W$. However, attention should also be paid to points on the boundary circle $\partial(\mathrm{H} / W)$-important boundary conditions, such as Dirichlet, Neumann and Robin boundary conditions, lie on this circle. In this section, we shall consider $\lambda_{n}$ as functions on $\partial(\mathrm{H} / W)$. Note that $\partial(\mathrm{H} / W)$ can be naturally viewed as the diagonal circle $S_{D}$ of $\mathrm{H}$, consisting of matrices of the form $e^{i \theta} I$.

It is known that the range of $\lambda_{n}$ on $\mathrm{U}(2)$ is the same as that of $\lambda_{n}$ on $\mathrm{H}$ [4], and the range is closely related to the eigenvalues of the Dirichlet boundary

condition. Since H is 2-dimensional, it's possible to determine this range by restricting $\lambda_{n}$ on $S_{D}$. In fact, we have

Theorem 5.1. The range of $\lambda_{n}$ on $\mathrm{U}(2)$ is the same as that of $\lambda_{n}$ on $S_{D}$. More precisely, if $\Lambda_{n, \kappa}$ is the $n$-th level- $\kappa$ curve on $\mathrm{H}$, then $S_{D}$ intersects $\Lambda_{n, \kappa}$ at a unique point.

Proof. The proof is based on Thm. 2.2 in [6], where in fact the level curve $\Lambda_{n, \kappa} \subset \mathrm{H}$ is characterized.

In [6], boundary conditions in $\mathrm{H}$ are written in the form of Eq. (1.2). It is easy to find that the diagonal of $\mathrm{H}$ corresponds to $\alpha, \beta$ 's satisfying $\alpha+\beta=\pi$. The level curve $\Lambda_{n, \kappa} \subset \mathrm{H}$ can be written as

$$
\left\{(\alpha, \beta) \in[0, \pi) \times(0, \pi] \mid \alpha=f(\beta), \beta \in J_{0}\right\},
$$


where the precise form of the interval $J_{0} \subset(0, \pi]$ depends on whether $\kappa>$ $\lambda_{n-1}^{D}$ or not, and the function $f$ is strictly increasing on $J_{0}$. So if $S_{D}$ intersects $\Lambda_{n, \kappa}$, the intersection point is unique. As for the existence of the intersection point, it can be derived easily from the argument of [6], cf. Fig.1 there.

Remark. It should be pointed out that in [6] there is another "diagonal" $\mathscr{C}$ in $\mathrm{H}$ (see Eq. (1.10) in [6]), which is different from ours. $\mathscr{C}$ corresponds to $\alpha, \beta$ 's satisfying $\alpha=\beta$, rather than $\alpha+\beta=\pi$. Thm. 5.1 does not hold when $S_{D}$ is replaced by $\mathscr{C}$.

Corollary 5.2. If $S_{D}$ is parameterized by $\beta \in(0, \pi]$ (so $\left.\alpha=\pi-\beta\right)$, then for each $n, \lambda_{n}$ as a function of $\beta$ is strictly increasing and continuous.

Proof. That $\lambda_{n}$ is continuous can be derived from Lemma 2.1 in [3]. For $\beta \in(0, \pi)$, the associated quadratic form is

$$
Q_{\beta}(y)=\int_{0}^{1}\left|y^{\prime}\right|^{2} d x+\int_{0}^{1} q(x)|y|^{2} d x-\cot \beta|\psi|^{2}, \quad y \in H^{1}
$$

and the strict monotonicity of $\lambda_{n}$ is a conclusion of the min-max principle and Thm. 5.1.

\section{Acknowledgemencts}

This study is supported by the Fundamental Research Funds for the Central Universities (2014B14514).

\section{References}

[1] M. Asorey, A. Ibort, G. Marmo, Global theory of quantum boundary conditions and topological change, Int. J. Mod. Phys. A, 20(2005), 10011025 .

[2] M.S.P. Eastham, Q. Kong, H. Wu, A. Zettl, Inequalities among eigenvalues of Sturm-Liouville problems, J. Inequalities Appl. 3(1999), 25-43.

[3] P.A. Binding, H. Volkmer, Interlacing and oscillation for Sturm-Liouville problems with seperated and coupled boundary conditions, J. Computational and Applied Mathematics 194(2006), 75-93. 
[4] Q. Kong, H. Wu, A. Zettl, Dependence of the n-th Sturm-Liouville eigenvalue on the problem, J. Differential Equations 156(1999), 328-354.

[5] Q. Kong, H. Wu, A. Zettl, Geometric aspects of Sturm-Liouville problems, I. Structures on spaces of boundary conditions. Pro. Royal Soc. Edinburgh 130A(2000), 561-589.

[6] X. Cao, Q. Kong, H. Wu, A. Zettl, Geometric aspects of Sturm-Liouville problems, III. Level surfaces of the $\mathrm{n}$-th eigenvalue, J. Computational and Applied Mathematics 208(2007), 176-193.

[7] J. Liu, Spectral Theory of Ordinary Differential Operators, in Chinese, Science Press, Beijing, 2009.

[8] K. Schmüdgen, Unbounded Self-adjoint Operators on Hilbert Space, Springer, GTM 265, 2012.

[9] M. R. Sepanski, Compact Lie Group, Springer-Verlag, Berlin Heidelberg, 2007. 